\title{
eNcentive: A Framework for Intelligent Marketing in Mobile Peer-To-Peer Environments
}

\author{
Olga Ratsimor \\ Department of Computer \\ Science and Electrical \\ Engineering, \\ University of Maryland \\ Baltimore County \\ 1000 Hilltop Circle, \\ Baltimore, MD 21250 \\ oratsi2@cs.umbc.edu
}

\author{
Tim Finin \\ Department of Computer \\ Science and Electrical \\ Engineering, \\ University of Maryland \\ Baltimore County \\ 1000 Hilltop Circle, \\ Baltimore, MD 21250 \\ finin@cs.umbc.edu
}

\author{
Anupam Joshi \\ Department of Computer \\ Science and Electrical \\ Engineering, \\ University of Maryland \\ Baltimore County \\ 1000 Hilltop Circle, \\ Baltimore, MD 21250 \\ joshi@cs.umbc.edu
}

\author{
Yelena Yesha \\ Department of Computer \\ Science and Electrical \\ Engineering, \\ University of Maryland \\ Baltimore County \\ 1000 Hilltop Circle, \\ Baltimore, MD 21250 \\ yeyesha@cs.umbc.edu
}

\begin{abstract}
In recent years, the growth of Mobile Computing, Electronic Commerce and Mobile Electronic Commerce has created a new concept of Mobile Electronic Marketing. New marketing models are being developed and used to target mobile users. Mobile environments introduces new challenges that need to be overcome by these marketing models in order to be successful and effective. This paper proposes a framework, called eNcentive, which addresses many of the issues that are characteristic of mobile environments. eNcentive facilitates peer-to-peer electronic marketing in mobile ad hoc environments. Our framework employs a intelligent marketing scheme, by providing users the capability to collect information like sales promotions and discounts. Users can propagate this marketing information to other users in the network. Participating users benefit from such circulation since businesses that originally created the promotions reward the active distributors with additional promotions and other compensations.
\end{abstract}

\section{Categories and Subject Descriptors}

H.4.3 [Communications Applications]: Miscellaneous; K.8 [Personal Computing]: Miscellaneous;

K.4.4 [Electronic Commerce]: Electronic data interchange (EDI)

\section{General Terms}

Electronic commerce

\footnotetext{
* This work was supported in part by NSF awards IIS 9875433, ISS 0209001 and CCR 0070802, and the Defense Advanced Research Projects Agency under contract F3060200-2-0591 AO K528.
}

\section{Keywords}

Electronic commerce, mobile commerce, trade and communication strategies, mobile electronic marketing, intelligent agents, collaborative eCommerce

\section{INTRODUCTION}

With the explosion of wireless technologies, it is becoming clear that mobile computing is slowly but surely becoming a dominant new culture [16]. People quickly get hooked onto the flexibility and autonomy that mobile devices can provide. Mobile users can no longer imagine their lives without their cellular phones, PDAs, MP3 players, digital cameras, etc. This mobile culture is in a fluidic state of nonstop evolution. New hardware, new software and new services are being developed and broadly used. Old applications and old services are being transformed to keep up with this mobile evolution. Along with new gadgets, applications and services, comes a set of new business models that address the needs of such mobile users. Mobile commerce is a new way to do business in this fast evolving mobile environment [17]. M-commerce is quite different form traditional electronic commerce. A new set of constraints is imposed on businesses and service providers that target mobile environments. Mobile devices have smaller screens, limited interface capabilities, restricted computational power and relatively short battery life [17]. Mobile users are less tolerant of irrelevant information and frequently have shorter attention span. Nomadic users demand applications and services that are context aware, personalized, address privacy and security $[17,16]$. M-commerce businesses, service providers and content providers must address these emerging issues in intelligent and creative ways. Mobile environments are particularly challenging for the businesses that generate their revenues thought advertising and marketing. In addition to the classical marketing problems that are common to other more conventional environments, these businesses have to deal with inpatient and demanding users that are equipped with restricted devices. Marketing information needs to be delivered in new intelligent ways so that the users find the information interesting, helpful and rewarding with minimal costs imposed on them and their devices. New marketing business models need to address individual user's personal preferences, user's location and temporal context along with 
privacy [18] and security issues [10].

Current marketing approaches use SMS (Short Message Service), MMS (Multi-media Messaging Services) and EMS (Enhanced Messaging Services) messages sent by merchants to the user's mobile phone. This type of marketing requires collaboration between multiple service providers. The mobile advertisement service provider have to take care of the preparation of the ad. Owners of the customer database have to define the target groups they will pursue. Mobile operators then have to select those of its customers that fit to the target group. The customer has to give the permission in advance to receive ads from the mobile operator. This is a long and complicated way to reach the consumers. Privacy violations could occur when users provide personal preferences to the content provider. Also preferences can change form hour to hour. They also depend on the user's context. It would be hard to maintain this dynamic preference list on remote database. Privacy and preference management would be clearly simplified if personal preferences were stored on the user's device (such preferences and profiles can be represented in DAML [1], RDF [2] or XML). However, this would complicate things for advertisers. Once user's preferences are moved to mobile device, the advertisers (in order to reach the customers) would have no other choice but to flood the network with advertisements sent to random customers. It is clear that this flooding model will not be successful even in a sort term. Other communication and marketing mechanisms need to be explored.

We believe that an alternate communication mechanism is peer-to-peer communication in mobile wireless environments. Frameworks like Numi [14, 11], MoGATU [13] and Proem [12] explore issues of peer-to-peer communications. Technology like Bluetooth [3], UPNP [4] and JINI [9] can facilitate device and service discovery (many devices like PDAs and mobile phones come equipped with Bluetooth). Marketing information can be injected in to such environments by services providers and marketing information distributors that are operating wireless basestations that are broadcasting promotions and advertisements. This broadcasted marketing information would be picked up by mobile devices that will come in range of the base station. The devices will consult with user's profile before storing the promotions and advertisements. Thus the promotions and advertisements will be transferred to the user's device in a private no intrusive matter. The problem with this approach is that broadcasts by a few basestations can reach only a small number of mobile users. Nevertheless, we believe that this problem can be solved by employing intelligent marketing model.

To address these issues we have developed a framework called eNcentive that facilitates peer-to-peer electronic marketing in mobile ad hoc environments. eNcentive employs a intelligent marketing scheme by collecting information like sales promotions and discounts, and marketing it to other users in the network, thereby deriving benefits when those other users utilize this information.

Consider a user equipped with a PDA running an eNcentive platform traveling though a geographical region populated by businesses (restaurants, cafes and drycleaners) that are actively broadcasting coupons, advertisements and other promotions to attract extra business. As the user passes by these businesses, the eNcentive platform running on the user's PDA actively collects/caches these coupons and promotions. These advertisements and coupons can be redeemed by this user at a later time at that business location or at any other service provider who honors those promotions. Alternatively, the user can employ the eNcentive platform to become a distributor of these coupons, promotions and advertisements. In this case, the platform starts to actively advertise coupons to other eNcentive peer platforms that the user passes by along the way. The peer platforms can cache these distributed advertisements and later redeem them with the business that honors these advertisements or likewise become another distributor. Thus a coupon can be pass from a user to another to yet another user before it is redeemed. To keep track of this chain, every coupon contains a list of platform IDs of every eNcentive platform that ever distributed this coupon. When a user decides to redeem a coupon and presents it to the business, the business after honoring the coupon stores the list of the platform IDs for future reference. Every participating business can choose to reward its most effective distributors with additional discounts or other rewards. For example, when a user redeems a coupon, the business can check its list of recently redeemed coupons and see how may times that user's eNcentive platform ID appears in those lists. The business can then reward the eNcentive user with additional discounts or upgrade the product or service the user was buying. This interaction thus is mutually beneficial to both the businesses and the users that distribute coupons on behalf of these businesses.

In the following sections we discuss related work, typical application scenario. We also present eNcentive agent based framework that we built as a prototype to demonstrate the feasibility of proposed approach. We conclude this paper by description of future work and summary and conclusions of work.

\section{RELATED WORK}

In recent years, a number of attempts were made to address marketing and advertising in mobile environments. In particular, there has been some work that targeted mobile phones. One such attempt was made by the Japanese video rental company, Tsutaya [5]. Tsutaya collected and maintained a database of its customers' musical and video preferences. With customers' consent, Tsutaya delivered highly personalized messages to the customers' mobile phones. The preferences where used to notify customers on their mobile phones of a release of new movies or new musical albums that matched customers' preferences. Tsutaya also sent out other promotional information like discounts on rentals and concert announcements that matched customers' personal preferences. Though very effective, the approach taken by Tsutaya has several draw backs. One of them is possible privacy concerns. Customers explicitly supply their personal information and personal preferences to the company. A valid concern of users for this sort of a model could be that this personal information is now at the full disposal of the company and can be used and even misused by it for unwanted spamming. Another drawback is that a user's preferences can change over time. In this model, it is up to the user to update the database to continue receiving relevant information. In addition, this approach does not address 
context awareness; a user might be disturbed at some inconvenient time or location.

Another advertising model that was adopted by a number of companies like Go2.com [6] involves adding advertisement information to the content that is being requested by the users. For example, if a user is requesting information about local restaurants he or she is presented with a wide spectrum of information including promotions. Users using Agents2Go [15] can receive static information like restaurant's address, phone number and menu along with frequently changing information like today's specials, waiting time and other restaurant promotions like coupons. This marketing approach has a great potential since, it takes into account users' context including time and location. It can also be personalized by asking users for their preferences ahead of time or even during the content delivery. However, along with the quality of information can come a concern of privacy again since the user's preferences are being sent to the server operated by the content provider. Also, it is going to be more difficult for smaller, less established businesses as passing users are not going to trust them with their personal information.

In October of 2002, an advertisement company Aerodeon [7] conducted a mobile marketing campaign for a popular confectionary brand in the UK. The campaign targeted UK teenagers though SMS messages. The promotions provided teens with the opportunity to become "squad leaders" and to create a unique "squad" name. Teens could invite as many friends as possible to join using SMS text messages. Each invitee in turn received a branded text message from the confectionary company asking them to recruit friends to join their unique "squad". UK's biggest "squad" won a year's supply of confectionaries. This marketing model actively engages users by necessitating collaboration and recruitment. However, success of this model is directly tied to the characteristics of the target groups and the type of promoted products. Active manual recruitment can, at times be intrusive and can violate privacy of both sending and receiving users. Scalability of the model can also be a major factor. Both sending and replying requires direct involvement of the users. Since users manually propagate the promotions to each other, they can be easily overwhelmed by active marketing environments. However, if the burden of solicitation, collaboration and marketing is shifted to devices like PDAs and mobile phones, the scalability and privacy problems can be avoided. If our personal devices are entrusted with our preferences and interests then they can make majority of evaluation and decisions our behalf.

In contrast to the above presented work, the eNcentive framework also addresses issues of privacy. Our framework allows users to store their profiles locally and does not require submission of any personal information to any centralized location. This inherently makes the framework privacy aware and more secure. Decisions, effecting the acceptance of the promotional information, can be made locally. This personal profile information can be presented to other peers based on the their reputation [8]. The frameworks' management component can be tied to the context monitoring component and to the personal preferences component. As a user's preferences evolve over time, the decision of infor- mation acceptance is automatically adjusted to reflect the new preferences. Thus, there is no need to maintain a remote profile for the user. This is especially very important to users' who depend on multiple content providers that are not linked and are not cooperating with each other. An argument can be made that locally storing profiles can be a factor for users with nonrestrictive profiles on mobile devices with limited computing capabilities. Mobile devices of such users could occasionally be weighed down by the incoming marketing information. We believe that using device profiles along with the user preferences can solve this problem. Another benefit of the eNcentive framework is that it also addresses location awareness since only neighboring peer devices are propagating information. It is logical to assume that the majority of the devices participating in information distribution are from that local geographical region and contain information that is relevant to the current location.

\section{TYPICAL SCENARIO}

The management of Jazz Cafe notices that in recent weeks they are getting fewer customers during evening hours. After some analysis, they come to the conclusion that this is a temporary phenomenon that can be overcome by additional advertisements. In attempt to attract more customers, Jazz Cafe issues electronic promotions. The promotion offers $10 \%$ off a cup of coffee bought after $5 \mathrm{pm}$.

The promotions are broadcast by the base station at the cafe. Jazz Cafe also has a set of loyal customers. One such customer is Bob. Bob, like many of us, is attached to his PDA (MH1) and carries it with him at all times. Bob's PDA is running the eNcentive platform. During Bob's morning visit to Jazz Cafe, Bob's MH1 receives and caches Jazz Cafe's e-promotion advertised by the base station. Once he leaves the cafe, $M H 1$ starts actively advertising this promotion to peer devises that it passes by. During the day, as Bob travels towards the pharmacy, he passes by Suzan who is equipped with a PDA (MH2) running eNcentive. MH2 receives Bob's broadcast and requests the promotion from MH1 as it matches Susan's drinking preference. MH1 signs the promotion with Bob's eNcentive ID and passes the promotion to Susan's MH2. Once Susan's PDA is out of Bob's range it starts actively advertising Jazz Cafe's promotion. In the afternoon, as Susan is on her way to the Fruit Mart, she passes by Jeff who is caring MH3. MH3 receives MH2 broadcast and requests the promotion (since Jeff is a coffee drinker). MH2 signs the promotion with Susan's $e N$ centive ID. At $6 \mathrm{pm}$, Jeff decides to take advantage of the Jazz Cafe promotion and visits the cafe. He presents his $10 \%$ off e-promotion as he purchases his cup of coffee. Jazz Cafe accepts the promotion and stores it in its eNcentive Database. Along with promotion, Jazz Cafe stores the $e N$ centive ID's of all the people that assisted in distributing this e-promotion. At $7 \mathrm{pm}$, Susan also visits the cafe. She uses her e-promotion to get the discount. Like Jeff's promotion, Susan's e-promotion is stored in the database along with the eNcentive ID's of the distributors. In addition to that, as she presents her promotion the Jazz Cafe, the eNcentive Server checks the eNcentive database and notices that she successfully assisted in distribution of one e-promotion (to Jeff). To reward Susan for her assistance, Jazz Cafe gives her $2 \%$ off in addition to the $10 \%$ off from the original e-promotion. 


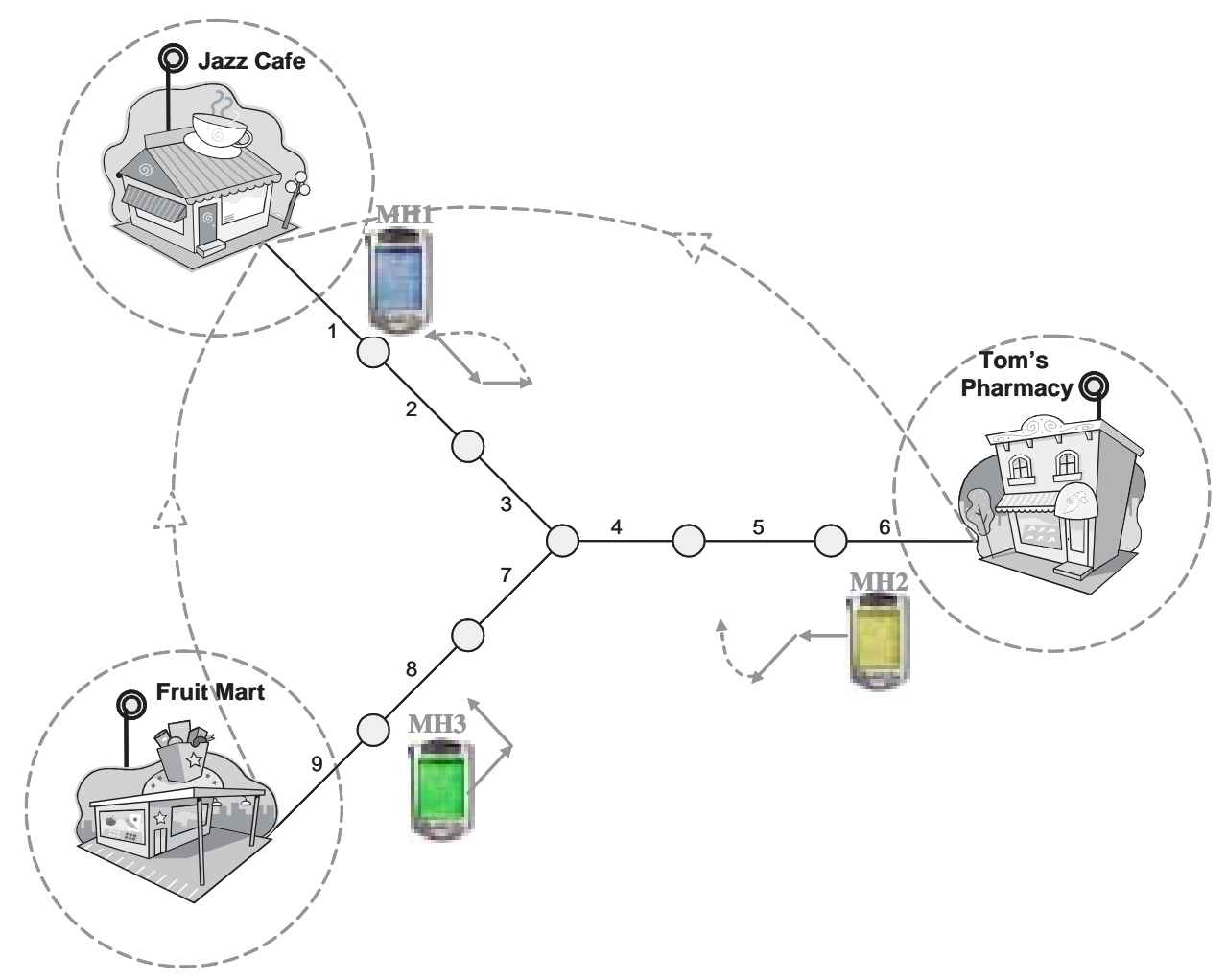

Figure 1: Device Architecture

So Suzan's final discount is $12 \%$ off. At $8 \mathrm{pm}$, Bob stops by Jazz Cafe to get his usual evening cup of coffee. He uses his e-promotion and receives additional $5 \%$ off on top of $10 \%$ guaranteed by e-promotion that he picked up in the morning. Bob's final discount is $15 \%$ since Bob eNcentive ID was present in Susan's and Jeff's e-promotions. At the end of the day, all parties have benefited in this interaction. Jazz Cafe has succeeded in attracting more customers through its promotion and because others distributed its promotions, the promotions were able to reach a wider audience, even those that were not in range of the cafe. Susan and Bob benefited through their additional discounts and Jeff now has found a new previously unknown coffee shop that is convenient and offers him discounts.

\section{ENCENTIVE FRAMEWORK}

The eNcentive framework is an agent based framework. By abstracting functionality into distinct agents, our framework is highly modular and loosely coupled. eNcentive was built as an application on top of an in-house agent framework called Numi $[14,11]$. Numi was built to address data management needs of mobile devices moving through a geographical region offering hybrid wireless support (combination of infrastructure based and ad hoc wireless technologies). The Numi framework envisions mobile devices moving through a geographical region populated with islands of cheap high-bandwidth network connectivity, close to access points, surrounded by regions of no connectivity or very expensive WAN connectivity. When mobile device are in range of an access point they are able to use the infrastructure and access information thought the high-speed connection.
When devices are away form access points they have to rely on other neighboring peer devices for their data needs. Numi acts as a perfect underlying framework since it provides a communication layer for eNcentive that can seamlessly work for both infrastructure based and ad hoc wireless networks. eNcentive reuses much of the existing functionality provided by Numi. Device and infrastructure discovery, location management, data communication and messaging, application management and logging are provided by Numi framework agents. eNcentive is implemented as an application running on top of the Numi runtime.

The eNcentive framework runs on both mobile devices and advertisers' portal (portal are access points managed by application servers). There are two configurations for the eNcentive framework: eNcentive Mobile Node Configuration and eNcentive Advertiser Configuration. Our modular design allows us to reuse same agents to implement these functionalities in both configurations.

\section{1 eNcentive Advertiser Con£guration}

The Advertiser Configuration is set up at the location of the business involved in marketing. It can be set up at the individual stores and shops or it could be used by an aggregate of multiple businesses. For example, a mall could run a single instance of this configuration on behalf all participating stores in that mall. The configuration consists of two eNcentive agents: eNcentive Ad Maker Agent and eNcentive Marketing Agent running on top of Numi Portal Configuration. 


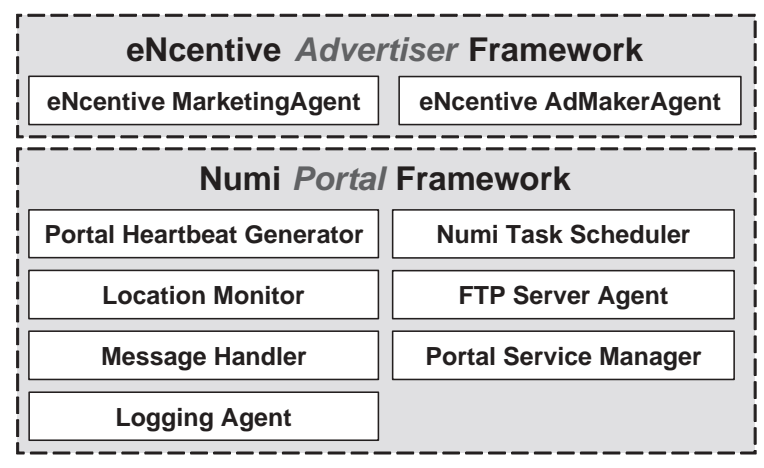

Figure 2: eNcentive Advertiser Configurations

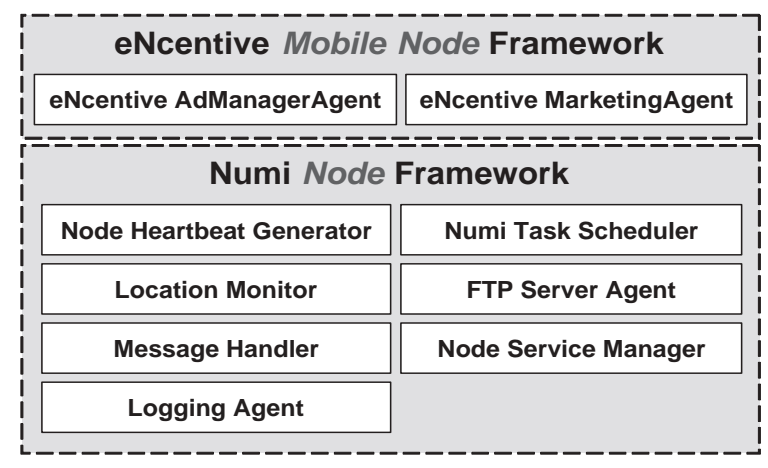

Figure 3: eNcentive Mobile Node Configurations
The eNcentive Ad Maker Agent provides advertisers with interfaces that allow them to create promotions and coupons. Promotions in eNcentive are objects that encapsulate information like discount information and details describing a marketing reward model that will be used as an incentive to active participants. For instance, a coffee shop e-promotion object can consist of discount information like " $10 \%$ off on any cup of coffee" and reward model of additional $1 \%$ off for every 15 successful references. Ultimately, the user will receive an additional $1 \%$ off (on top of original $10 \%$ off) for every 15 people that will cite this user as part of their reference chain. As the e-promotion gets passed around form one mobile device to another it accumulates the list of eNcentive platform IDs. This ID's represent chains of references (users that were instrumental in the promotion distribution) that are later used to reward the active distributors. Current implementation of eNcentive allows creation of coupons that can have a set of restrictions. For example, promotions can have start time and end time. For instance, coupons can be generated and distributed during the week days but the discount is valid only on the weekends. Another restriction that can be imposed on the coupon is number of times it is passed from one person to the other. Currently, we assume that coupons and promotions are copied from one user to the other, with the original user continuing to retain possession of the coupons and promotions. Thus, it is possible that the number of e-promotions in the network is greater than originally created. We are currently working on adding a functionality that would restrict duplication and insure the controlled proliferation of these coupons. Another possible restriction that we are considering is adding location radius to the promotions. If the e-promotion is marketed too far away from the business then that e-promotion becomes invalid. Radius restriction can be used in cases of multiple branches of business spread over several geographical regions. If the promotion is honored only by the branches of a particular region then this promotion should be invalidated outside the that region.

Once an e-promotion is created, it is given to eNcentive Marketing Agent. This agent is responsible for scheduling of advertisement release. The Marketing Agent is essentially in charge of the advertising policy. It specifies how frequently an e-promotion should be broadcasted by the advertising platform. This allows periodic and controlled injection of marketing information into the network.

\section{2 eNcentive Mobile Node Confguration}

Broadcast advertisements and promotions are picked up by the mobile devices running eNcentive Mobile Node Configuration. This configuration consists of two eNcentive agents running on top of Numi Node Platform: eNcentive Marketing Agent and eNcentive Ad Manager Agent. To reuse the modular functionality of eNcentive, we utilized the Marketing Agent used in eNcentive Advertiser Configuration. The job of Marketing Agent running on Mobile Node Configuration is to distribute advertisements that are approved by the local Ad Manager Agent.

The eNcentive Ad Manager Agent performs a number of tasks on the mobile node. This agent collects, organizes and maintains promotions. The collection function of the agent is linked to the user profiles and current user context. Once Numi platform notifies Ad Manager Agent about incoming advertisements, the agent consults the user profiles and makes a determination whether to collect or to ignore the advertisement. Current implementation of our framework employs relatively simple profiles described in XML. We are currently working on a more sophisticated implementation were profiles are implemented in RDF [2]. The eNcentive Ad Manager Agent also maintains and organizes already collected promotions. The promotions are kept in a lightweight data structure that is linked to the user interface. The promotions are also categorized based on their start and end times. Our data structure also insures that the information is displayed in the appropriate order. The Ad Manager Agent also is able to organize promotions by location. In particular, the Ad Manager agent can notify users that he or she is in range of a business that accepts the coupons held by the user. Thus, if the promotion is about to become valid, a user has a choice of remaining in his current location and being able to take advantage of the promotion. The eNcentive Ad Manager Agent is also in charge of responding to the peer requests. That is, if a device $A$ is interested in the promotion that is being advertised by the device $B$ then Ad Manager Agent of $A$ request the Ad Manager Agent of $B$ to forward the promotion. When the request is granted the Ad Manager Agent of $B$ inserts $B$ 's platform ID into the requested promotion and then forwards this to $A$.

Current design of the eNcentive framework employs a push model. The advertisements are actively broadcast through out a network. This is clearly not the only model that can 


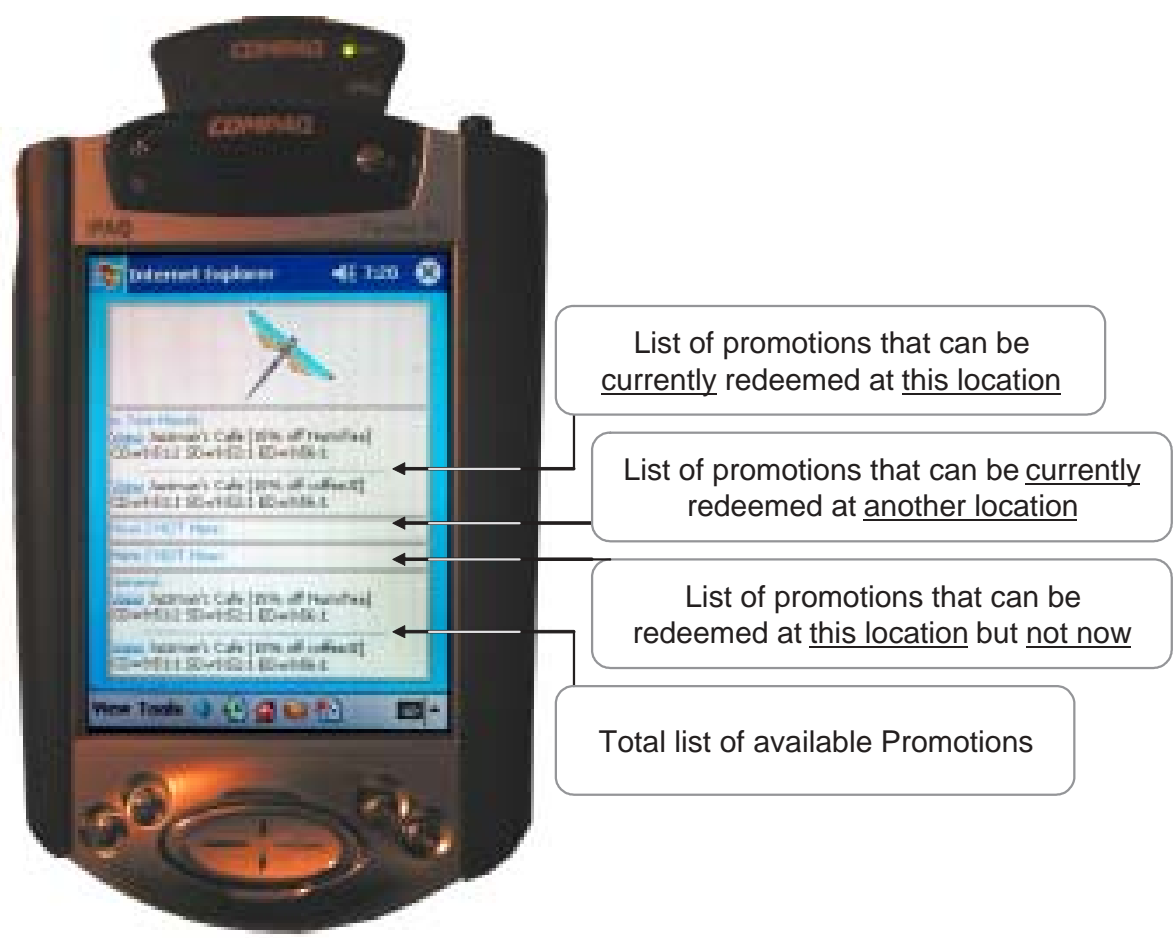

Figure 4: Web Interface for eNcentive Mobile Node Configuration

be used. A pull model can also work well in mobile peer-topeer environment. Mobile devices that are interested in the marketing information can query other peer device in the neighborhood. Other hybrid approaches are also possible.

\section{PROTOTYPE IMPLEMENTATION}

As a demonstration of our proposed approach, we have developed a working prototype. We have implemented our prototype using Java programming language. Our platform is installed on three PCs and three iPAQs. The PCs run the eNcentive Advertiser Configuration on top of Numi Service Portal Platform and the iPAQs run eNcentive Mobile Node Configuration on top of a Numi Mobile Node Platform.

All devices used were equipped with 802.11 b wireless LAN cards. The iPAQs were running the Jeode Embedded Virtual Machine. Each Advertiser was also running a Tomcat Apache Servlet Engine. To simulate the mobility of the devices (moving in range and out of range of each other) we divided each an imaginary geographical region into nonoverlapping cells. Each cell has a unique cell ID. Mobile nodes were able to communicate with each other only if they are in the same cell. Numi platform filtered out all messages that did not match a device's cell ID. By using this notion of cells, we are able to simulate neighborhoods and by changing a device cell ID; its neighborhood could be changed thereby simulating movement. We have developed an additional simulation component called the Mobility Coordinator. Using this, control messages can be sent to any device to change its current cell ID.

\subsection{Prototype Setup}

We have set up three Advertisers (PCs): Jazz Cafe, Fruit Mart and Tom's Pharmacy. All three advertisers were periodically (every 15 seconds) broadcasting coupons with discounts. Jazz Cafe Advertiser followed a reward model described by the formula and graph in Figure 5. This reward model allowed gradual growth of rewards for up to $70 \%$ off. Other two Advertisers followed a threshold reward model. For the first ten references, users got $5 \%$ off; for the ten to thirty references, users got $10 \%$ off; and for thirty and up user got $20 \%$ off. Other models are possible.

Three iPAQs: Node1, Node2 and Node3 were running $e N$ centive Mobile Node Configuration. Node 1 was put into range of Jazz Cafe Advertisers. Once Node1 picked up a coupon form the Advertisers, it was moved out of its range. Node 1 then was then put in range with Node2. Once Node2 heard the cafe promotions from Node1, it requested this promotion. Before the promotion was transferred, Node1 inserted its eNcentive ID into this promotion. Then Node2 was put in range with Node3. Node3 requested the promotion from Node2. Once the promotions became valid (promotions had start and stop time), nodes were one by one moved into range of the advertiser. The coupons were redeemed and active participants (Node1 and Node2) were rewarded with extra discounts. Node1 was the greatest benefactor since it referred Node2 and Node3. Node 3 received no additional discounts on top of the original promotion.

\section{CONCLUSION AND FUTURE WORK}

In this paper we have presented a novel model for intelligent marketing in the mobile environments. We have built a prototype framework, called eNcentive, that allows users, 


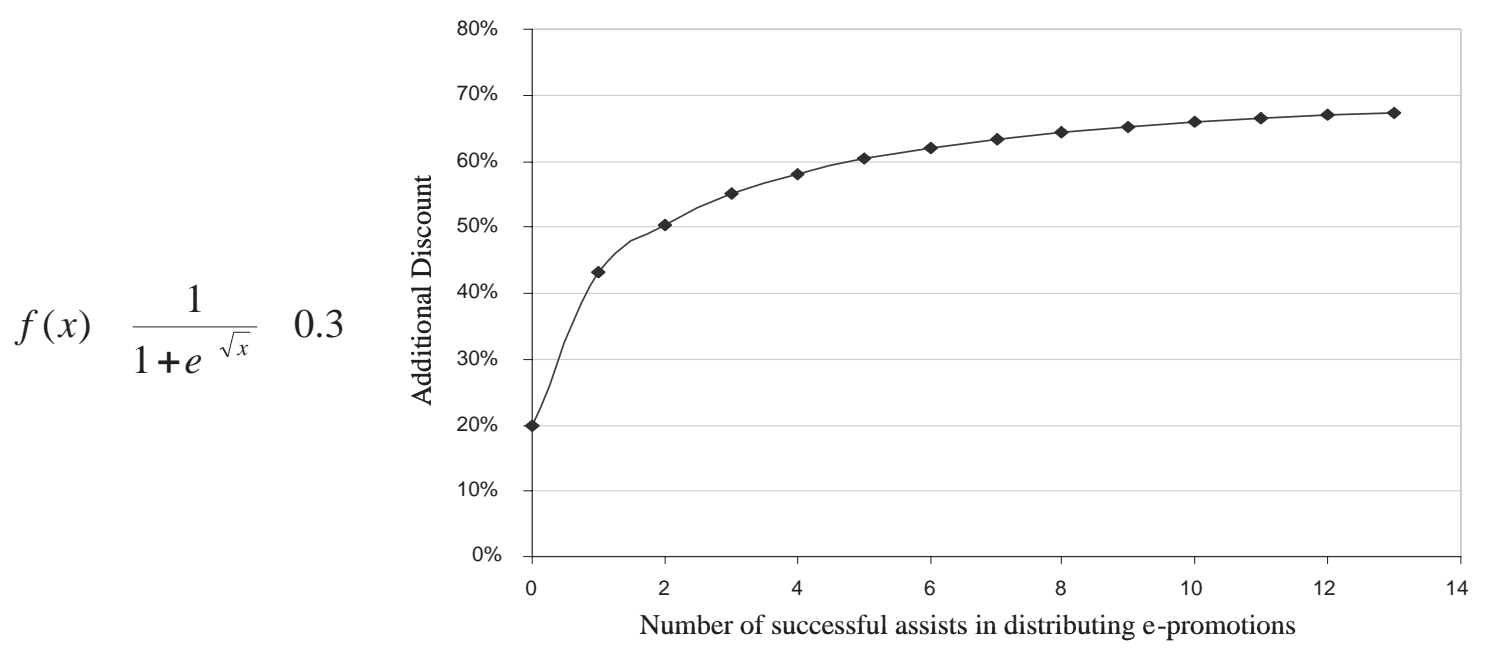

Figure 5: Example of eNcentive Reward Model Used by Advertisers

equipped with mobile devices, to collect advertisements, promotions, coupons and other marketing information that is being broadcast by businesses. These devices, later, can rebroadcast these advertisements to other peer devices. Each time an advertisement is passed from a device to another peer device, the platform ID of the broadcasting device is inserted into the advertisement. When the advertisement or promotion is presented as a form of payment for an item or a service, the business application server stores the IDs of all the devices that were involved in distributing this promotion. The owners of these devices are rewarded at a later time. Reward models can vary; however, it is clear that users and devices should be rewarded in accordance with the success rate that they have achieved in effectively distributing the advertisements and promotions. We are currently working on expanding functionalities our framework. We are adding more sophisticated device and user profiling. We are also experimenting with interfaces for collecting user preferences locally on their mobile devices. In particular we are looking at possibilities of the device learning user's preferences over time. We are also looking into making the framework more location and context ware by linking it with a user's calendar. We are currently exploring other reward models like aggregation of multiple businesses supporting each other by giving cross promotions.

We believe this form of distribution of marketing information can be very effective in mobile environments. Individuals can choose to collect the advertisements that are of most interest to them and in the same time, they can profit from such collection by redistributing the advertisements to their peers. This form of marketing is, flexible, personalized, private, location and context aware, and most importantly, it lets user be in control of information coming in and out if his or her device. Also, as there is no central user preference database, our model is less intrusive on users' privacy thereby making it more appealing from a user point of view. We believe our model can be very successful once it is accompanied with an attractive and effective reward model.

\section{REFERENCES}

[1] DARPA Agent Markup Language, World Wide Web, http://www .daml.org.

[2] Ora Lassila, Ralph R Swick, Resource Description Framework (RDF) Model and Syntax Specification, W3C Recommendation, 22 February 1999. http: //www.w3.org/TR/1999/REC/rdf-syntax-19990222.

[3] Bluetooth White Paper, World Wide Web, http://www.bluetooth.com/developer/whitepaper.

[4] UPnP White Paper, World Wide Web, http://upnp.org/resources.htm.

[5] Tsutaya Inc., World Wide Web, http://www.tsutaya.tv/.

[6] Go2 Inc., World Wide Web, http://www.go2online.com/.

[7] Aerodeon Limited., World Wide Web, http://www . aerodeon. com/press.html.

[8] Lethin, R.: Chapter 17: Reputation. Peer-to-Peer: Harnessing the Power of Disruptive Technologies ed. Andy Oram, O'Reilly and Associates (2001).

[9] K. Arnold, A. Wollrath, B. O'Sullivan, R. Scheifler, and J. Waldo. The Jini specification. Addison-Wesley, Reading, MA, USA, 1999.

[10] L. Kagal, J. Undercoffer, F. Perich, A. Joshi, and T. Finin. A security architecture based on trust management for pervasive computing systems, 2002.

[11] S. B. Kodeswaran, O. Ratsimor, A. Joshi, T. Finin, and Y. Yesha. Using peer-to-peer data routing for infrastructure-based wireless networks. In IEEE International Conference on Pervasive Computing and Communications (PerCom 2003), Fort Worth, TX, USA, March 2003. 
[12] G. Kortuem, J. Schneider, D. Preuitt, T. G. Thompson, S. Fickas, and Z. Segall. When peer-to-peer comes face-to-face: Collaborative peer-to-peer computing in mobile ad hoc networks. In Proceedings 2001 International Conference on Peer-to-Peer Computing, Aug, 2001.

[13] F. Perich, S. Avancha, D. Chakraborty, A. Joshi, and Y. Yesha. Profile driven data management for pervasive environments. In 3th International Conference on Database and Expert Systems Applications (DEXA 2002), Aix en Provence, France, September 2002.

[14] O. Ratsimor, S. B. Kodeswaran, A. Joshi, T. Finin, and Y. Yesha. Combining infrastructure and ad-hoc collaboration for data management in mobile wireless networks. In Workshop on Ad-hoc Communications and Collaboration in Ubiquitous Computing Environments held in conjunction with ACM 2002 Conference on Computer Supported Cooperative Work, New Orleans, Louisiana, USA, November 2002.

[15] O. Ratsimor, V. Korolev, A. Joshi, and T. Finin. Agents2go: An infrastructure for location-dependent service discovery in the mobile electronic commerce environment. In ACM Mobile Commerce Workshop held in conjunction with MobiCom 2001., Rome, Italy., July 2001.

[16] H. Rheingold. Smart Mobs: the next social revolution. Perseus Publishing, September 2002.

[17] N. Sadeh. Mobile Commerce: New Technologies, Services and Business Models. Wiley, 2002.

[18] J. Xiaodong, J. Hong, and J. Landay. Approximate information flows: Socially-based modeling of privacy in ubiquitous computing, 2002. 